\title{
Ballistic electron transport in hybrid ferromagnet/two-dimensional electron gas sandwich nanostructure: Spin polarization and magnetoresistance effect
}

\author{
Y. Wang, ${ }^{1, a)}$ N. F. Chen, ${ }^{1,2}$ Y. Jiang, ${ }^{3, a)}$ and X. W. Zhang ${ }^{1}$ \\ ${ }^{1}$ Key Laboratory of Semiconductor Material Sciences, Institute of Semiconductors, Chinese Academy of \\ Sciences, Beijing 100083, China \\ ${ }^{2}$ National Laboratory of Microgravity, Institute of Mechanics, Chinese Academy of Sciences, \\ Beijing 100080, People's Republic of China \\ ${ }^{3}$ School of Materials Science and Engineering, University of Science and Technology Beijing, \\ Beijing 100083, China
}

(Received 21 September 2008; accepted 28 October 2008; published online 7 January 2009)

\begin{abstract}
We have theoretically investigated ballistic electron transport through a combination of magnetic-electric barrier based on a vertical ferromagnet/two-dimensional electron gas/ferromagnet sandwich structure, which can be experimentally realized by depositing asymmetric metallic magnetic stripes both on top and bottom of modulation-doped semiconductor heterostructures. Our numerical results have confirmed the existence of finite spin polarization even though only antisymmetric stray field $B_{z}$ is considered. By switching the relative magnetization of ferromagnetic layers, the device in discussion shows evident magnetoconductance. In particular, both spin polarization and magnetoconductance can be efficiently enhanced by proper electrostatic barrier up to the optimal value relying on the specific magnetic-electric modulation. (C) 2009 American Institute of Physics. [DOI: 10.1063/1.3041477]
\end{abstract}

\section{INTRODUCTION}

The feasibility of spin polarization and spin filtering within a two-dimensional electron gas (2DEG) has been proposed by Papp and Peeters ${ }^{1}$ based on the combination of hybrid magnetic-electric barrier structure, which can be experimentally realized by patterning ferromagnetic (FM) materials on top of modulation-doped semiconductor heterostructures. In such systems, the stray field of FM stripe constitutes a spatial nonhomogeneous magnetic barrier to manipulate locally the motion of electron within 2DEG channel, and accounting for this spin-field interaction, spin polarization in this device was expected. Unfortunately, for single FM stripe with in-plane magnetization along the transport direction, no spin polarization ${ }^{2-4}$ should occur if the antisymmetric stray field $B_{z}$ is only considered. Physically, when electron tunnels through a pair of antisymmetric magnetic barriers, any spin polarization caused by a positive magnetic barrier will be compensated completely by an opposite effect induced by a negative one. The detailed analysis on system symmetry ${ }^{5}$ has also suggested that, since the Hamiltonian of this system is invariant under the transformation of $\hat{T} \hat{R}_{x} \hat{R}_{y}$, where $\hat{T}$ is the time-reversal operator and $\hat{R}_{x}\left(\hat{R}_{y}\right)$ is the reflection operator, the resulting electron transmission is identical for both spin channels and thus zero spin polarization in this system occurs in the linear response regime. Therefore, in order to obtain the finite spin polarization, one must break the structure symmetry governed by the operator $\hat{T} \hat{R}_{x} \hat{R}_{y}$.

To date, several methods ${ }^{5-9}$ have been proposed to

\footnotetext{
${ }^{a)}$ Authors to whom correspondence should be addressed. Electronic addresses: ywang@semi.ac.cn and yjiang@mater.ustb.edu.cn.
}

achieve the finite spin polarization based on analogous structures by breaking this intrinsic symmetry. Of particular interest here is the proposal to construct a full symmetric magnetic barrier as initially suggested by Jiang and Jalil ${ }^{9}$ by means of depositing two stripes both on top and bottom of 2DEG plane. Besides the spin polarization effect, the additional magnetoresistance (MR) effect is also expected in this configuration. However, due to the accumulation of transverse vector potential arising from the symmetric stray field, there is a net increase in transverse momentum of electron before and after tunneling, ${ }^{10-12}$ implying the shift of propagating direction of electron after tunneling in comparison to the incident one. Moreover, the enhancement of small spin polarization by increasing the number of symmetric barriers used $^{13}$ will lead to low transmission probability. Thus the combination of magnetic barrier with averaging zero accumulation of vector potential is particularly beneficial for the realization of spin transport. In this work we will remove this unexpected accumulation by taking into account the magnetic field omitted in previous investigations, and the behaviors of external-tunable spin polarization as well as MR effect are revealed based on an alternative configuration.

\section{THEORETICAL MODEL}

Our system is schematically depicted in Fig. 1(a), where a 2DEG moving in the $x-y$ plane is sandwiched by two asymmetric metallic FM stripes with in-plane magnetization (along $x$-direction). Especially, two stripes are designed deliberately flush left and the strength of out-of-plane stray field emanated from magnetic stripes is denoted by $B_{1}\left(B_{2}\right)$ for bottom (top) one, respectively. To study MR effect, we assume that the bottom FM stripe of width $L$ is a harder 

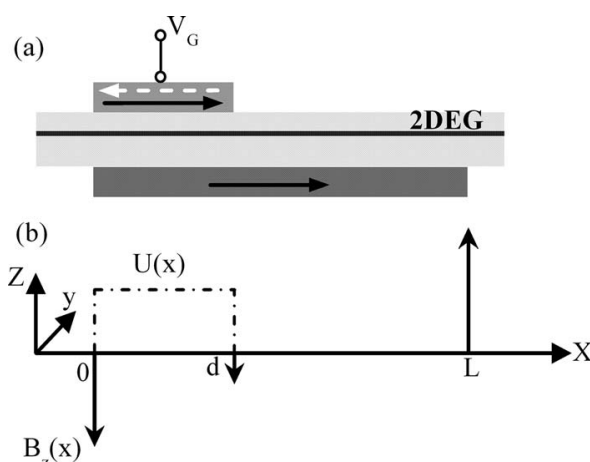

FIG. 1. Schematic of device structure (a) and theoretical model (b) employed for electrostatic barrier and stray field profile under parallel configuration.

magnetic layer in comparison with top one of width $d$, allowing the switch of relative magnetization between stripes from parallel (P)/antiparallel (AP) to AP/P configuration by external magnetic field. In addition, applying a voltage $V_{G}$ on the top stripe, an electrostatic modulation is incorporated. Qualitatively, we approximate the exact stray field as delta function and use rectangle barrier to describe electrostatic term as shown in Fig. 1(b). Hence electric barrier as well as the integrated profiles of stray field can be, respectively, expressed as $U(x)=U \theta(x) \theta(d-x)$ and $B_{z}(x)=B_{1}[\delta(x-L)$ $-\delta(x)]+\gamma B_{2}[\delta(x)-\delta(x-d)]$, where $\gamma= \pm 1$ denotes the configuration of stripes with the positive (negative) sign for $\mathrm{P}$ (AP) alignment, $U$ is the height of electric barrier, and $\theta(x)$ is the well-known Heaviside step function.

Here, in order to clarify the spin polarization induced only by the out-of-plane field $B_{z}$, we neglected the in-plane stray field $B_{x}$ because of its symmetric characteristic. In the framework of single particle effective mass approximation, the Hamiltonian of system can be written as

$$
\hat{H}=\frac{(\hat{P}+e \vec{A})^{2}}{2 m^{*}}+U(x)+\frac{e g^{*}}{2 m_{0}} \frac{\sigma_{z} \hbar}{2} B_{z}(x),
$$

where $m_{0}$ is the free electron mass, $m^{*}$ is the electron effective mass, $g^{*}$ is the effective Landé factor, $\sigma_{z}=+1 /-1$ corresponds to spin up/down electron, respectively, $\hat{P}$ is canonical momentum operator, and magnetic vector potential, in the Landau gauge, is given by $\vec{A}=\left[0, A_{y}(x), 0\right]$ with $A_{y}(x)=$ $-B_{1} \theta(x-d) \theta(L-x)+\left(\gamma B_{2}-B_{1}\right) \theta(x) \theta(d-x)$. Here for convenience, we express all quantities in dimensionless units: (1) the magnetic field $B_{z}(x) \rightarrow B_{0} B_{z}(x)$; (2) the vector potential $A_{y}(x) \rightarrow B_{0} \ell_{B} A_{y}(x)$; (3) the coordinate $x \rightarrow \ell_{B} x$; (4) the energy $E \rightarrow \hbar \omega_{c} E\left(=E_{0} E\right)$, by introducing two characteristic parameters: the cyclotron frequency $\omega_{\mathrm{c}}=e B_{0} / \mathrm{m}^{*}$ and the magnetic length $\ell_{B}=\sqrt{\hbar / e B_{0}}$, where $B_{0}$ is some typical magnetic field. For example, for $B_{0}=0.1 \mathrm{~T}$, this gives the reduced units $E_{0}$ $=0.48 \mathrm{meV}$ and $\ell_{B}=81.3 \mathrm{~nm}$ if the effective mass is taken for InAs system as $m^{*}=0.024 m_{0}$.

Since the system is translation invariant along the $y$-direction, the total wave function describing the transport of electron with incident energy $E$ can be written as $\psi(x, y)$ $=e^{i k_{y} y} \phi(x)$, where $k_{y}$ is the $y$-component of wave vector and $\phi(x)$ can be further solved exactly from reduced onedimensional Schrödinger equation,

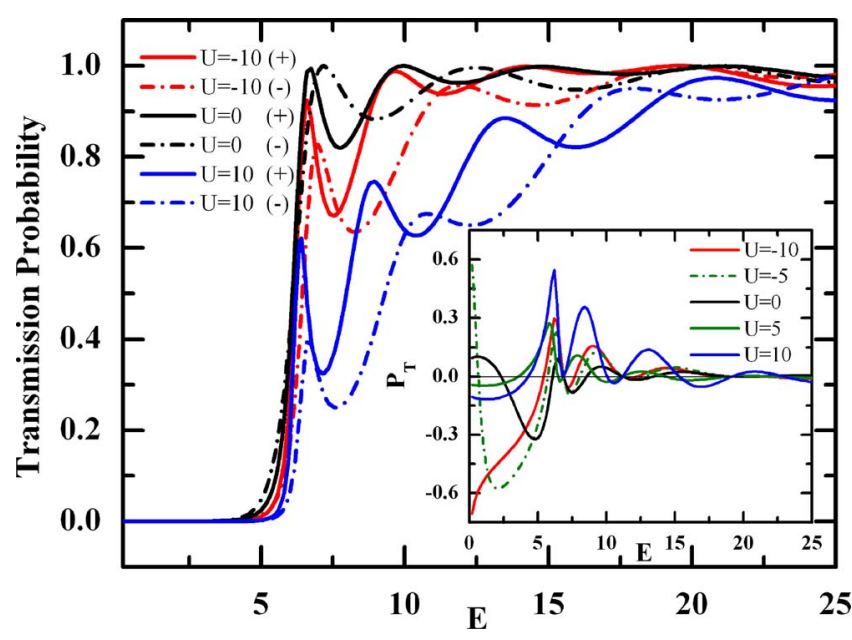

FIG. 2. (Color online) Spin-related transmission probabilities of parallel configuration taking for different electric barriers as a function of incident energy $E$ with an incident angle $\theta=\pi / 6$. In the inset, the corresponding transmission polarization $P_{T}$ is shown.

$$
\left[\frac{d^{2}}{d x^{2}}+U_{\mathrm{eff}}(x)\right] \phi(x)=0,
$$

with effective potential $U_{\text {eff }}(x)=2[E-U(x)]-\left[k_{y}+A_{y}(x)\right]^{2}$ $-\left(g^{*} m^{*} / 2 m_{0}\right) \sigma_{z} B_{z}(x)$. Based on the above equation, by means of standard transfer matrix method, one can calculate spin-resolved transmission probability $T\left(E, k_{y}, \sigma_{z}\right)$, from which other ballistic transport properties such as conductance and MR can be further determined. For instance, spinresolved conductance under zero temperature is easily deduced in a ballistic regime from Landauer-Büttiker formula, ${ }^{14}$

$$
G_{\sigma_{z}}\left(E_{F}\right)=G_{0} \int_{-\pi / 2}^{\pi / 2} T\left(E_{F}, \sqrt{2 E_{F}} \sin \theta, \sigma_{z}\right) \cos \theta d \theta,
$$

where $\theta$ is incident angle relative to transport direction, $E_{F}$ is the Fermi energy of 2DEG, and $G_{0}=e^{2} m^{*} v_{F} L_{y} / h^{2}$ is the reduced unit of conductance with electron Fermi velocity $v_{F}$ and length $L_{y}$ of the system in the $y$-direction.

\section{RESULTS AND DISCUSSION}

In our numerical simulation, to see more clearly spindependent effect, we take material parameters of 2DEG for InAs system (i.e., $g^{*}=15, m^{*}=0.024 m_{0}$ ) due to its large effective $g$ factor. Unless the special clarification, we assume that the parameters of top stripe are $d=0.4$ and $B_{2}=2$, and the corresponding values for bottom one are selected as $L=2$ and $B_{1}=5$. In Fig. 2, we plot schematically the spin-resolved transmission probability as a function of incident energy for different electric barriers, where electron incident angle is selected as $\theta=\pi / 6$. It is clearly seen that, starting from a threshold energy, all spectra of transmission display the pronounced oscillation with the increasing energy due to the presence of quasibound state above the quantum barrier/well induced by hybrid magnetic-electric suppression. The threshold energy, beyond which the evident transmission probability is observed, mainly depends on the accumulation of vector potential and incident angle, showing well-confirmed 


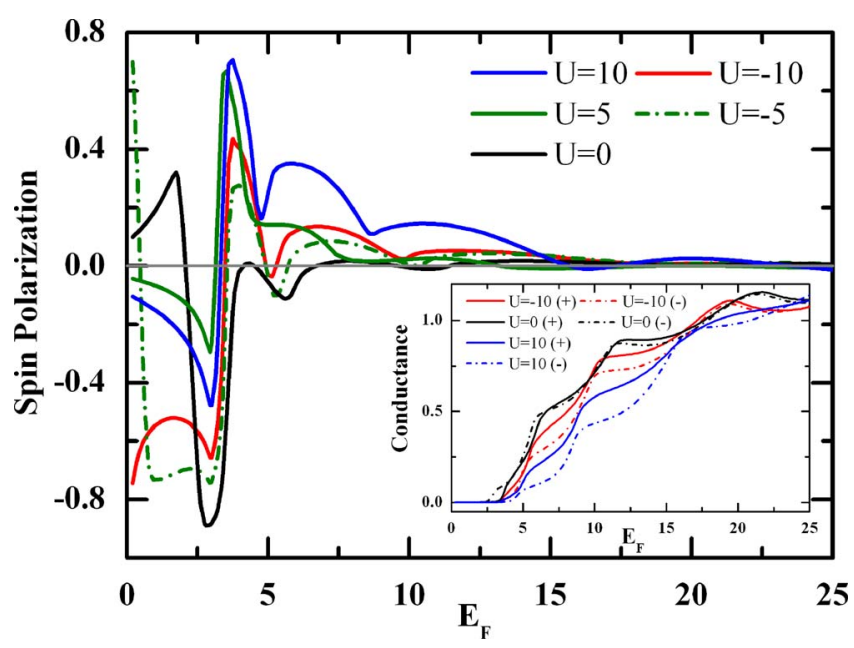

FIG. 3. (Color online) Spin polarization as well as spin-resolved conductance (inset) as a function of Fermi energy of 2DEG under various electrostatic suppressions.

wave-vector filtering ${ }^{15}$ effect. Particularly, the splitting of transmission into two spin-resolved branches indicates the considerable existence of spin polarization within the considered structure. In the inset, the corresponding energy $E$-dependence of transmission polarization $P_{T}(E, \theta)$, defined as $\quad P_{T}(E, \theta)=[T(E, \theta,+1)-T(E, \theta,-1)] /[T(E, \theta,+1)$ $+T(E, \theta,-1)]$, is shown. Obviously, with the elevating electron energy $E$, there is a rapid reduction in the amplitude of oscillatory transmission polarization as a consequence of its finite strength of stray fields, the modulation of which are weakened for large energy. By applying the gate-controllable voltage, the spin-related transmission presents a quite distinct response relying on the sign of electric barrier. Concretely, due to its strong dissipation effect, electron transmission for positive electric barrier is intensely blocked in comparison with the cases for dissipationless negative electric barrier and vanishing electrostatic modulation, giving rise to the large decrease in transmission probability. Prominently, the transmission polarization of system under consideration is efficiently enhanced by positive electric barrier within realistic energy scope.

Since the intrinsic two-dimensional tunneling process, the obtained $P_{T}$ strongly depends on incident angle $\theta$, which is experimentally trouble measurable. To remove this inconvenience and reflect this spin asymmetric effect on measurable quantities such as conductance $G_{ \pm}(E)$, we evaluate in our work the spin polarization of device as $P_{G}=\left[G_{+}(E)\right.$ $\left.-G_{-}(E)\right] /\left[G_{+}(E)+G_{-}(E)\right]$. In Fig. 3, this spin polarization under the parallel configuration is calculated for different electric barriers. Due to the oscillatory transmission, all spectra of spin polarization exhibit evident oscillation with the increasing Fermi energy. For small $E_{F}$, there is a giant difference between two spin channels because of the intense modulation coming from stray field, leading to the very high polarization, whereas the blocking transmission within this forbidden region makes the conductance undetectable, as shown in the inset of Fig. 3. Beyond certain threshold energy conductance for both channels shows rapid increase, while the spin asymmetric effect shrinks significantly as a conse-

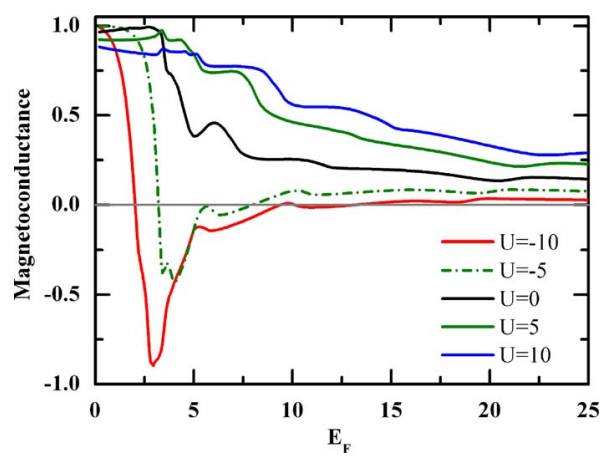

FIG. 4. (Color online) Magnetoconductance vs the increasing Fermi energy $E_{F}$ for different electric barriers.

quence of weakening magnetic-electric suppression, resulting in the very reduction in spin polarization. Especially, there are several kinks in all conductance- $E_{F}$ curves related to the contribution of the quasibound states within the structure. By incorporating electric barriers, spin polarization is efficiently modified. For example, if a positive electric barrier $U=10$ is induced, the typical polarization for Fermi energy $E_{F}=10.6$ can be remarkably enhanced up to $15 \%$ from an invisible one corresponding to the absence of electrostatic term $U=0$. Therefore, we have confirmed the existence of finite spin-polarization and spin-filtering effects in considered structure. Physically, although the induced field $B_{z}$ is antisymmetric for each single stripe, the integrated stray field emanated from both stripes as we demonstrated here might not be antisymmetric, and thus the breaking system symmetry governed by $\hat{T} \hat{R}_{x} \hat{R}_{y}$ just assures the presence of expected spin polarization. Besides, since there is no accumulation of transverse vector potential before and after electron tunneling, the further improvement of this weak polarization by means of constructing multiple-period structure is promised.

Besides the feasibility of spin-filtering effect in hybrid device, the MR effect as observed in conventional FM multilayers ${ }^{16}$ is also expected. Traditionally, when the relative magnetization of adjacent magnetic layers is switched, the quite distinct spin-dependent scattering leads to the striking variation in electric resistance. However, in our case, when the magnetization configuration is switched, there is a substantial change in the effective potential and the resulting transmission is quite different, thus the obtained MR effect might be spin independent. ${ }^{17-19}$ Here we calculate this spinless MR ratio in terms of magnetoconductance as $\Delta G=\left(G_{\mathrm{P}}\right.$ $\left.-G_{\mathrm{AP}}\right) /\left(G_{\mathrm{P}}+G_{\mathrm{AP}}\right)$, where $G_{\mathrm{P}} / G_{\mathrm{AP}}$ denotes the total conductance for P/AP alignment, respectively. In Fig. 4, $E_{F}$-dependent $\Delta G$ is presented for different electrostatic modulations. It is obviously seen that all $\Delta G$ show remarkably degradation with the increasing $E_{F}$ because of weakening magnetic-electric confinement. Moreover, the incorporation of positive electric barrier has effectively improved the magnitude of MR ratio, while for a negative barrier the MR ratio shows evident reduction within the realistic energy region and even changes its sign. For very small $E_{F}$, there is an abrupt variation both in the magnitude and the sign of $\Delta G$, indicating the presence of a specific resonant state related to AP configuration. Based on the same reason mentioned 


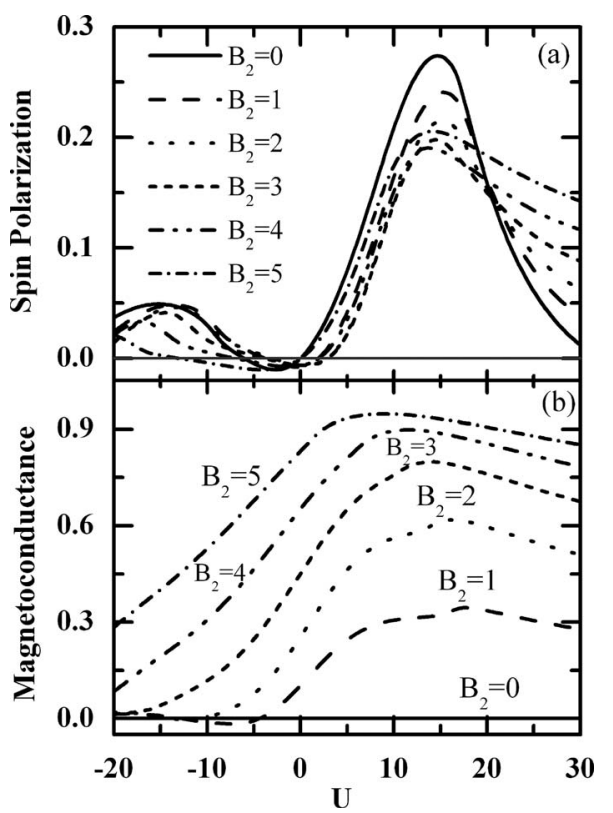

FIG. 5. $U$-dependent spin polarization (a) and magnetoconductance (b) for different stray fields of top stripe.

above, all $E_{F}$-dependent $\Delta G$ have pronounced fine kinks, more visible for a stronger confinement. For Fermi energy $E_{F} \approx 10, \Delta G$ for pure magnetic barrier is only about $25 \%$, which can be significantly improved by electrostatic barrier up to $46 \%$ for $U=5$ and $56 \%$ for $U=10$. Even for a higher Fermi energy $E_{F} \approx 25$, the MR ratio near $30 \%$ is still expected by incorporating an electric barrier $U=10$.

Having seen both spin polarization and magnetoconductance can be considerably influenced by external electrostatic modulation, it is interested to know to what extent these quantities can be tuned by electric barrier. As for example, we schematically depict these quantities versus the variation in electrostatic barrier in Fig. 5, where the Fermi energy of 2DEG has been selected for illustration as 10. Evidently, both spin polarization and MR ratio can be effectively enhanced by a proper electrostatic barrier up to the optimal value, which strongly depends on the specific magneticelectric barrier condition. Beyond this critical barrier, both quantities display large degradation since the dominant electrostatic modulation in this case is inherent configuration and spin independent. Notice that, for magnetic strengths $B_{2}=0$ and 5, the obtained parallel spin polarization is zero for pure magnetic barrier as shown in Fig. 5(a) because the integrated field is antisymmetric in these situations. As for $\Delta G$ depicted in Fig. 5(b) is concerned, the typical value for considered structure can be efficiently enhanced from $25 \%$ of pure magnetic modulation up to $62 \%$ by including an electric barrier $U=16$, showing twofold strong improvement. For a stronger magnetic field $B_{2}=5, \Delta G$ larger than $90 \%$ is also expected. Generally, the stronger magnetic confinement is accomplished, the higher MR ratio is expected.

It should be noted that for a real InAs materials, whose typical electron densities are of the order of $10^{12} \mathrm{~cm}^{-2}$, which gives large Fermi energy $E_{F} \approx 42$, corresponding to $E_{0}=2.4 \mathrm{meV}$ with typical magnetic field $B_{0}=0.5 \mathrm{~T}$. For such high incident energy, magnetic-electric modulation dis-

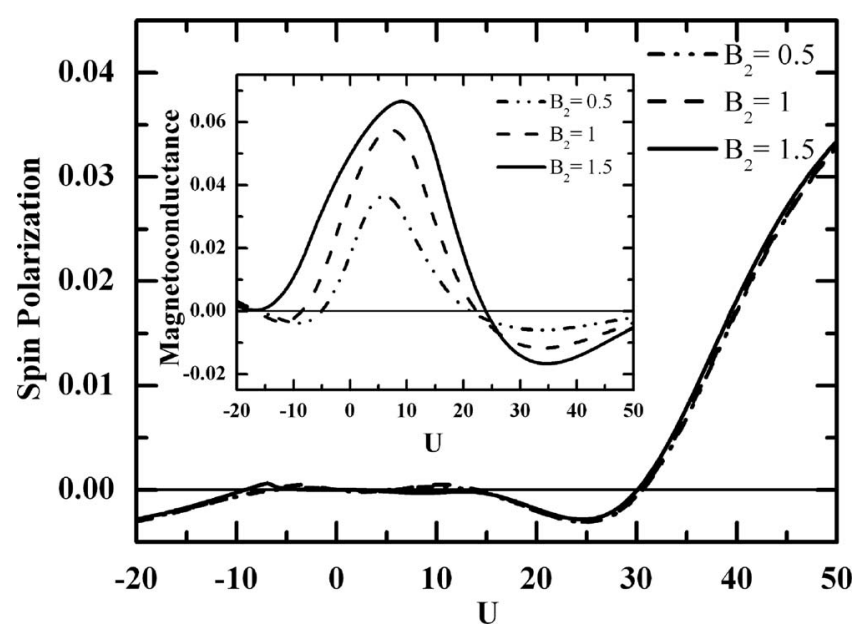

FIG. 6. Spin polarization and magnetoconductance (inset) as a function of electric barrier for a realistic InAs system.

cussed above only plays a minor role, leading to very small spin polarization and magnetoconductance as shown in Fig. 6 , where the magnetic strength of bottom stripe is fixed at $B_{1}=1.5$ for the reduced unit $B_{0}=0.5 \mathrm{~T}$, while the strengths for top one are taken as $B_{2}=0.5,1$, and 1.5 , respectively. Due to its tiny strength in comparison to the incident energy, the expected spin polarization is trouble resolvable when the stray field of top stripe is modified. However, there is a remarkable improvement in magnetoconductance induced by the stronger field of top stripe, indicating its less spindependent feature. Typically, both polarization and magnetoconductance are not more than $10 \%$ for this weak magnetic modulation. Therefore, to achieve higher spin polarization and MR ratio, additional efforts must be paid to realize much stronger magnetic-electric suppression.

\section{CONCLUSIONS}

In conclusion, we have theoretically investigated in ballistic regime the spin-dependent transports based on a vertical FM/2DEG/FM sandwich structure, where the vanishing accumulation of transverse vector potential and the breaking system symmetry governed by operator $\hat{T} \hat{R}_{x} \hat{R}_{y}$ have been realized. Numerical results have confirmed the existence of finite spin polarization as well as spinless MR effect. In particular, both quantities can be efficiently enhanced by the optimal electrostatic barrier up to the maximum value determined mainly by the magnetic-electric barrier condition.

\section{ACKNOWLEDGMENTS}

Y. Jiang was supported by the NSFC (Grant Nos. 90607020 and 50571021) and National Basic Research Program of China (Grant No. 2007CB936202).

${ }^{1}$ G. Papp and F. M. Peeters, Appl. Phys. Lett. 78, 2184 (2001).

${ }^{2}$ G. Papp and F. M. Peeters, Appl. Phys. Lett. 79, 3198 (2001).

${ }^{3}$ Y. Jiang, M. B. A. Jalil, and T. S. Low, Appl. Phys. Lett. 80, 1673 (2002).

${ }^{4}$ H. Z. Xu and Y. Okada, Appl. Phys. Lett. 79, 3119 (2001).

${ }^{5}$ F. Zhai, H. Q. Xu, and Y. Guo, Phys. Rev. B 70, 085308 (2004).

${ }^{6}$ M. B. A. Jalil, J. Appl. Phys. 97, 024507 (2005).

${ }^{7}$ F. Zhai and H. Q. Xu, Appl. Phys. Lett. 88, 032502 (2006).

${ }^{8}$ B. Wang, Y. Guo, X. Y. Chen, and B. L. Gu, J. Appl. Phys. 92, 4138 
(2002).

${ }^{9}$ Y. Jiang and M. B. A. Jalil, Phys. Status Solidi B 235, 157 (2003).

${ }^{10}$ G. Papp and F. M. Peeters, Phys. Status Solidi B 241, 222 (2003); Y. Jiang and M. B. A. Jalil, ibid. 241, 224 (2004).

${ }^{11}$ M. W. Lu, Solid State Commun. 134, 683 (2005).

${ }^{12}$ Y. Guo, J. H. Qin, X. Y. Chen, and B. L. Gu, Semicond. Sci. Technol. 18, 297 (2003).

${ }^{13}$ M. B. A. Jalil, S. G. Tan, T. Liew, K. L. Teo, and T. C. Chong, J. Appl. Phys. 95, 7321 (2004).
${ }^{14}$ M. Büttiker, Phys. Rev. Lett. 57, 1761 (1986).

${ }^{15}$ A. Matulis, F. M. Peeters, and P. Vasilopoulos, Phys. Rev. Lett. 72, 1518 (1994).

${ }^{16}$ M. N. Baibich, J. M. Broto, A. Fert, F. Nguyen Van Dau, F. Petroff, P. Etienne, G. Creuzet, A. Friederich, and J. Chazelas, Phys. Rev. Lett. 61, 2472 (1988).

${ }^{17}$ F. Zhai, Y. Guo, and B. L. Gu, Phys. Rev. B 66, 125305 (2002).

${ }^{18}$ M. W. Lu and L. D. Zhang, J. Phys.: Condens. Matter 15, 1267 (2003).

${ }^{19}$ G. Papp and F. M. Peeters, J. Phys.: Condens. Matter 16, 8275 (2004). 